\title{
A concise review of nanomaterials for drug delivery and release.
}

\author{
Alfonso Toro-Córdova ${ }^{\mathrm{a}}$, Beatriz Sanz ${ }^{\mathrm{a}, \dagger}$ and Gerardo F. Goya ${ }^{\mathrm{a}, \mathrm{b},{ }^{*}}$ \\ ${ }^{a}$ Institute of Nanoscience of Aragon, University of Zaragoza, Zaragoza, Spain; ${ }^{b}$ Condensed Matter Physics Department, \\ Faculty of Sciences, University of Zaragoza, Zaragoza, Spain;
}

\begin{abstract}
This review provides an updated vision about the recent developments in the field of drug vectorization using functional nanoparticles and other nanovectors. From the large number of these nanotechnology-based drug delivery systems (DDS) that emerge nearly every week, only a tiny fraction reach a pre-clinical or clinical phase study. In this report, we intend to provide contextual information about those nanocarriers and release methods that have shown the best outcomes at in vitro and in vivo experiments, highlighting those with proven therapeutic efficiency in humans. From silica-based porous nanoparticles to liposomes or polymeric nanoparticles, each one of these nanosystems has its advantages and drawbacks. We describe and discuss briefly those approaches that, in our criterion, have provided significant advancements over existing therapies at the in vivo level. This work also provides a general view of those commercially available nanovectors and their specific area of therapeutic action.
\end{abstract}

Keywords: Drug Release, Drug Delivery Systems, Nanoparticles, Nanosystems, Therapeutic Drugs, In vivo applications.

\section{INTRODUCTION}

The idea of controlled drug release, as opposed to immediate release, refers to the control of the temporal and spatial presentation of a therapeutic molecule in the body. Sustained-release dosages aim to maintain the drug availability within specific therapeutic windows of concentration and time. The use of micro- and nanomaterials as drug carriers to improve the drug release is a rather old concept reported already in the ' 80 s when micellar solutions were developed for controlled release formulations.[1] Each controlled-release system has to be designed not only with specific attention on the chemistry of the drug to be administrated but also considering the specificities of the targeted organ (e.g., crossing physiological barriers, protect from quick elimination, etc.). The advent of nanotechnology to the field of medicine made possible novel surface-modified nanocarriers for drug targeting and controlled release. This review aims to provide an succinct revision of those recently developed drug release methodologies that, in our opinion, have shown the best performance in animal models or clinical applications. We start with a description of some types of carriers of use in drug vectorization, followed by few in vitro successful experiments with those nanosystems. Finally, we appraise the state of the art regarding those commercial products of nanocarrier-based drug delivery systems (DDS) already approved by the regulatory agencies.

\section{TYPES OF CARRIERS}

\subsection{Liposomes}

Lipidic carriers are suitable for in vivo applications due to their similitude with biological structures that veils the immune system and result in very long circulation times after intravenous administration. The low immune response against liposomes is partially due to a reduced phagocytosis, a mechanism demonstrated at the in vitro level using blood THP-1 monocyte cells in human plasma.[2] Lipid-based nanosystems can be divided into non-lamellar and lamellar types. The latter are usually obtained from direct assembly of amphiphilic building blocks, while the former include liposomes, cubosomes and spongosomes. Liposomes were reported about 50 years ago,[3] but it was only two decades later that their ability as DDS for therapeutic applications was reported.[4] Liposomes can be described as lipid vesicles composed of an aqueous core surrounded by lipid bi- or multilayers, with average sizes around $50-200 \mathrm{~nm}$. The amphipathic character of phospholipids make possible to encapsulate either hydrophilic molecules at the aqueous core or hydrophobic drugs within the lipid bilayers of the liposomes.[5]

\footnotetext{
${ }^{\dagger}$ Also at nB nanoscale Biomagnetics S.L., Zaragoza, Spain.

*Address correspondence to this author at the Department of Condensed Matter Physics, Faculty of Sciences, University of Zaragoza, 50018, Zaragoza, Spain; Tel/Fax: ++34-876-555-362, +34-976-762-776; E-mail: goya@unizar.es
} 


\subsection{Extracellular vesicles}

An interesting strategy for drug delivery recently developed is the use of extracellular vesicles (EVs) of different intracellular origin.[6] The highly efficient cell uptake mechanisms of EVs allow boosting the delivery doses for not only synthetic drugs, but also RNAs or proteins. EVsbased strategies have the advantage of an easy overcome of biological barriers.[7] Additionally, it has been reported that curcumin-loaded EVs increase drug's solubility, stability and protection against lipopolysaccharide (LPS)-induced microglial activation that causes brain inflammation. [8] The different biogenesis of EVs determines the final biological function, and can be described with three main classes: exosomes, micro-vesicles and apoptotic bodies.[9] In general, all types of EVs are composed of a surrounding lipid bilayer, and their sizes can be found to vary widely in the literature from $\approx 30 \mathrm{~nm}$ to about 2 microns in diameter. Among these, exosomes are perhaps the most used because their ability to degrade themselves after fusion with lysosomes at the intracellular space. It has been reported that exosomal encapsulation of doxorubicin and paclitaxel are effective for targeting brain tumours.[10] Exosomes are released into the extracellular environment through an endo-lysosomal pathway, after fusion of multi vesicular endosomes with the cell surface (see Figure 1). It has been demonstrated, at both in vitro and in vivo levels that exosomes are mediators of intercellular communication through transfer of messenger molecules.[11]

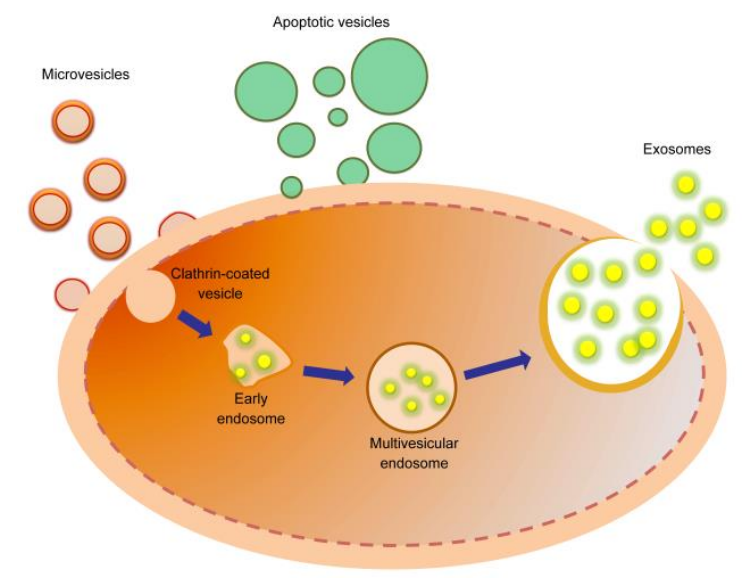

Figure 1. Schematic view of the pathway formation of extracellular vesicles, including exosomes, microvesicles, and apoptotic vesicles. In many cases, extracellular vesicles have signalling functions involved in different biological processes like antigen presentation, activation of cell surfaces through major histocompatibility complex (MHC) molecules, or transporting miRNAs.

\subsection{Nanoparticles}

The use of functionalized nanoparticles (NPs) as carriers for controlled vectorization and release of drugs is based on the wide spectrum of physical properties of the NPs core, which offers different ways of on-site drug release by application of a remote physical stimulus such a light, magnetic fields, electromagnetic radiation or ultrasonic waves.[12-16] Each one of these strategies has its pros and cons, and the choice among them depends on the particularities of the specific clinical situation to be solved.[17]

\subsubsection{Metallic Nanoparticles}

The developments in synthetic chemistry of the last decades have provided many new nanostructured materials with new physicochemical properties. These properties can originate either from the larger surface/volume ratio of nanometer-sized materials, or from subtler size-effects of physical nature (e.g., plasmonic resonance, superparamagnetism, etc.). Some successful applications reported in biomedicine have been connected with the use of semiconductor nanocrystals [18], gold nanoparticles and silver nanoshells [19],[20]. Gold nanoparticles possess unique photodynamic properties related to the absorption of near-infrared radiation, a mechanism useful for both diagnosis and therapy. These applications have been discussed in detail elsewhere, notably the comprehensive works of Dykman et al.[21] and Panchapakesan et al.[22]. Several noble metals ( $\mathrm{Pt}, \mathrm{Pd}, \mathrm{Ag}, \mathrm{Au}$, etc.) are being explored as new, cutting-edge nanostructured biocatalysts due to their abundant exposed active sites and highly accessible surfaces.[23] Moreover the interaction of metallic $\mathrm{Au}$ particles with membranes when heated with light has been recently studied.[24]. More rare materials like Rhodium nanoparticles, synthesized by chemical reduction on polymeric micelle templates, have shown better electro catalytic oxidation of methanol than the bulk counterparts [25], a property that could be adapted to modify cellular biocatalytic pathways, provided Rh-toxicity can be kept at low values.

\subsubsection{Magnetic Nanoparticles}

Magnetic nanoparticles (MNPs) have been projected for remotely controlled release since at least since 1969.[26] The possibility of remote magnetic actuation on MNPs by dc magnetic fields, and their coupling with ac magnetic fields to release heat have triggered a plethora of nanodevices in biomedicine, many of them with great success.[27],[28] Although these applications are well-known and standardized, it is remarkable that the physical mechanisms that govern drug release under alternate magnetic fields is still a matter of controversy, i.e., whether a mechanical rather than a thermal effect is actually acting locally when radiofrequency (RF) magnetic fields are applied. This is partially related to the fact that measuring local temperatures (i.e., at the nanometer scale) at the particle surroundings has proven extremely difficult.[29],[30] In any case, the improvement of the release rates when an EMF of a given frequency is applied has been clearly demonstrated experimentally.[31] The control of drug release with EMF operates in different types of carriers and reservoirs; the most used being surface-coated NPs. However, other thermoresponsive carriers and reservoirs have been reported to be effective, such as thermoresponsive ferrogels,[32] thermomagnetic polymer films,[33] and polymer films with embedded NPs for synergic uses of drug 
release and thermal tissue ablation. Regarding MNPs, the selection of magnetic materials of the magnetic cores has been based primarily on biocompatibility requirements, making the iron-oxide based MNPs the most extensively reported so far. A second, more labile criterion for choosing the magnetic core relies on those intrinsic magnetic parameters like saturation magnetization, magnetic anisotropy, Curie temperature, etc. that may improve the magnetic response.

In a recent work using MNPs in a liposomal formulation, Guo et al.[34] were able to delivery antineoplastic drugs to the local tumour environment in animal model. Moreover, they used two concurrent physical stimuli (IR light and radiofrequency) to trigger the release bursts of the cytotoxic drugs within the liposomes, achieving promising therapeutic results in vivo.

Reviews on the different types of metallic and magnetic materials used to produce nanoparticles for drug delivery can be found elsewhere.[17] Here, we will mention only that for the purposes of drug delivery/release nanosystems translated into clinics, manufacturing these systems under good manufacturing practice (GMP) standards is technically achievable. On the other side, as nicely described in Ref.[35], the steps to clinical implementation can be difficult and lengthy (see also Section 5.2 below) and it is along these stages that roughly a $95 \%$ of the designed nano-devices fail to comply the stringent clinical requirements. A prospective analysis of the experimental works of the last years indicated that most of the successful applications at the in vivo level are based on the use of multi-stimulus DDS like the previously described. The emerging picture is that any single activation method by itself would be less efficient than the combination of two or more of them, as in the successful case of MNPs and liposomes. These emerging strategies have not been yet fully explored.

\section{RELEASE MECHANISMS}

\subsection{Solid Drugs and Solid Matrices}

When a solid dosage form is required for specific therapeutic situation, it is essential to warrant that drug dissolution (release from the solid matrix) occurs in an appropriate manner. Indeed, these drug release kinetic profiles are mandatory from regulatory authorities. The quantitative analysis of the values obtained in dissolution / release tests are usually numerically simulated using models that express the dissolution rate as a function of the chemical parameters for each dosage form. The models are often empirically derived from previous experiments that constitute a databank, although in simple cases theoretical models are available.[36] Drug release rates from solid dosage forms have been described by kinetic models based on the time-dependence of the dissolved amount of drug $\mathrm{Q}(\mathrm{t})$. These models are usually referred as zero-order, first-order, etc, reflecting the functional dependence of $\mathrm{Q}$ with time. The quantitative interpretation of the values obtained in the dissolution assay is facilitated by the usage of a generic equation that mathematically translates the dissolution curve of each pharmaceutical dosage form. In some cases, that equation can be deduced by a theoretical analysis of the process, as for example in zero-order kinetics.

\subsection{Solid Drugs in Water-Soluble Polymers}

Many therapeutic drugs have a too-high toxicity profile for a direct administration in vivo. For this reason, many molecules intended for clinical applications require a modified formulation in order to minimize toxicity, as well as to improve efficacy of release kinetics. One of the proposed ways to achieve these goals has been the use of polymeric microspheres and nanospheres that could control the in vivo drug release profiles. Solid dispersions in water-soluble carriers have been successful in this sense, especially by modifying solid-to-liquid release rates of highly hydrophobic drugs.[37]

The concept of a 'solid dispersion' covers any dosage of one or more active ingredients dispersed in a biologically inert solid matrix.[38] [39] The mechanisms behind the observed improved release kinetics when compared to solid-solid systems are not yet completely understood, but there is some consensus regarding the key roles of the crystallographic structure and dissolution properties on the final release rates. [40] Some models of carrier-controlled kinetics have been proposed to calculate the release rates in terms of the concentration profile at the polymer layer adjacent to the solid surface. [41]

A reported alternative to control the release kinetics is the use of cyclodextrins in polymeric nanospheres and hydrogels. [42] Cyclodextrins are cyclic oligosaccharides that have the ability to form non-covalent complexes, changing the physicochemical properties of the carriers. However, only few reports on the actual efficacy have been published,[43] while none in vivo tests have been reported to the best of our knowledge.

\subsection{Coated Tablets}

Perhaps the best-known method used to improve high-density formulations is to coat tablets with functional films. Specifically, tablet coatings can be used to achieve drug stabilization and to modify/delay drug release when oral delivery forms are required.[44] A group of successful systems developed last decade for colon-specific delivery, which reported good specificity in vivo included pressurecontrolled colon delivery capsules (PCDCs) and colonic DDS based on pectin and galactomannan coating. The latter DDS was reported by Lee et al.[45] and consisted of a tablet coated with two specific polysaccharides (pectin and galactomannan). The solubility of this coating formulation was found to depend on the $\mathrm{pH}$, being insoluble at $\mathrm{pH} \geq 7$ but dissolving fast at those $\mathrm{pH} \leq 7$ typical of intestinal fluids, although the authors did not discuss any model for this $\mathrm{pH}$ dependence.

\subsection{Remote Radiofrequency-Triggered Release}

Non-invasive strategies for remotely triggering drug release have been proposed mainly for liposomal-based nanovectors. [46] These strategies can be based on different triggering stimuli, including (but not restricted to) enzymatic [47], 
temperature [48], light [49], magnetic fields [50] and ultrasound. [51] Of special clinical interest is the control of drug release profiles on demand by a remote alternating magnetic field of low frequency (i.e., at the low part of the RF spectrum, $100-800 \mathrm{kHz}$ ), because of the deep penetration of these waves without noticeable interaction with biological tissues. In order to load the MNPs with the therapeutic drug to be delivered, several coating/functional approaches are possible. Iron oxide MNPs attached to porous silica have been reported as an efficient way to store chemical payloads and release them upon triggering using a RF field.[52] In the same work, an increase of desorption rates under applied RF magnetic fields has been reported. Remotely triggered DDS for cancer applications rely deeply on recent improvements in synthesis of MNPs and MNPs-based polymeric materials like ferrogels [53] or magnetoliposomes (MLPs) [54]. The uniqueness of remote triggering by low-frequency magnetic fields relates with is large penetration depth of these waves into the human body without interaction with the healthy organs or tissues. The price to pay, on the other hand, is the need of delivering a 'heating agent' i.e., the magnetic nanoparticles to the target site to be heated.

\subsection{Remote Ultrasound-Triggered Release}

Ultrasound is another of the non-invasive, remote stimulus that can be focused on targeted sites for triggering drug release. Since ultrasonic waves have the additional effect of increasing the permeability of blood-tissue barriers and cell membranes, they can be used synergistically to control release kinetics.[55] So far, the liposomal formulations are the nanocarriers with best responsiveness to ultrasound stimulation. Acoustically active liposomes with an average size of $800 \mathrm{~nm}$ have already been used as contrast agents for ultrasound image enhancement[56],[57], while magnetoliposomes offer the additional possibility of magnetic resonance imaging protocols.[58] In the case of acoustic imaging, the main mechanism seems to be associated to air vesicles encapsulated within liposomes, which determine their acoustic activity.[59] Moreover, these liposomes have the potential to respond to ultrasound stress by releasing their contents.

\section{TESTING DRUG DELIVERY/RELEASE IN VITRO}

The main sequence of in vitro events involving targeting, internalization and drug release is common to almost any DDS, since target sites for most therapies are located at the intracellular space. As an example, cationic liposomes possess the ability to form complexes (lipoplexes) with nucleic acids through electrostatic interaction, which determines their transfection efficiency. Improved internalization of DDS is usually achieved through surface functionalization with cell penetrating peptides or membrane permeable ligands.
Once at the intracellular space, different DDS are designed to react to the cytoplasm conditions to stimulate drug release. In such strategies, the nanocarrier is usually designed to be sensitivity to cell temperature; $\mathrm{pH}$ changes; redox potential and/or elevated reactive oxygen species (ROS) levels (see Figure 2). The different physical stimuli used to remotely activate release mechanisms include radiofrequency (RF), near-infrared light (NIR) and ultrasound (US), already discussed in the previous section.

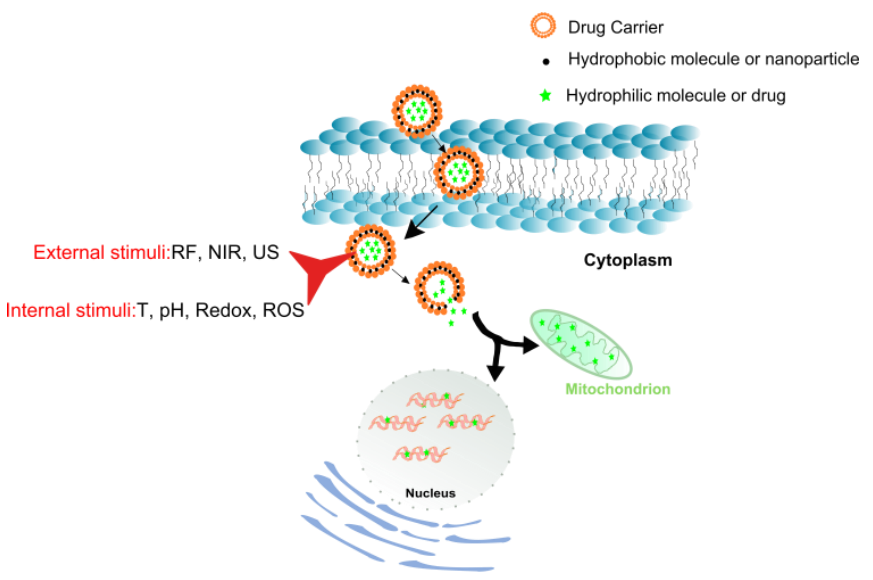

Figure 2 Schematic illustration of drug carrier internalization into the intracellular space and its activation by different external or internal stimuli.

In vitro release tests are recognized as a significant piece of pharmaceutical information since in vitro dosage form can influence the release kinetics.[60] Under certain conditions it can be a good estimator for assessment of bioequivalence. Most of the nanosystems described in the previous section have transited different processes towards final clinical or biomedical applications. While many of them have been tested in vitro for a variety of cell lines, only a relatively small number of these proof-of-concept nanovectors have been tested in animal models (see below). In the following subsections we review the most representative approaches tested so far in primary cell types, and some that have gone all the way through the in vitro tests to the clinical approval.

Polymer-based carriers are among the systems extensively tested in vitro.[61] The presence of primary, secondary, and/or tertiary amine groups provide effective, linkable surfaces with negatively charged molecules of biological interest (e.g., nucleic acids). Several cationic polymers have been tested, and Olden et al.[62] reported that a subset of sunflowershaped polymer NPs could mediate gene transfection on both cultured and primary cells with efficiencies up to $50 \%$ in the Jurkat human T cell line. Additionally, these NPs were reported to show minimal concomitant toxicity (> 90\% viability), opening possible ways to optimize primary T-cell transfection conditions including activation time, cell density, DNA dose, culture media, and cytokine treatment.

Another approach that has consensus regarding its efficacy in vitro is the use of ferritin-based nanocarriers. Ferritin is an iron-storage protein consisting of 24 subunits that selfassemble to form spherical nanocages of around $12 \mathrm{~nm}$ in 
diameter with an interior cavity of $8 \mathrm{~nm}$.[63] Chemical groups $(\mathrm{N}$ - and $\mathrm{C}$ - terminal sites) present at the outer surface provide the possibility to bind different compounds. Zhang and coworkers presented the use of apoferritin as delivery agent, with reports of low cytotoxicity and low immune response.[64] Translocation of apoferritin cage allowed the encapsulation of doxorubicin (Dox) inside the cavity forming a stable complex (H-Dox). Due to the chemical and size properties, H-Dox is able to pass the blood-brain barrier in vivo. Zhang et al. showed its potential using cerebellar organotypic cultures and suggested the possibility of using a double chambered cage containing different targets. This idea was further developed by Kin et al.[65] who described the formation of double-chambered ferritin cage NPs in which the first N-terminal chamber is loaded with peptide and the second C-terminal chamber to be loaded with a tumourtargeting pro-apoptotic peptide and the fluorescent protein GFP, respectively.

Related to the above approach, micellar nanocarriers have been also used at the in vitro level to test their multifunctional potential. For example, Jing et al. [66] presented a micellar nano-platform capable of simultaneously showing high cell penetration and nuclear targeting through $\mathrm{pH}$-triggered surface charge reversal. The results reported on mice bearing 4T1 breast tumour showed enhanced cellular internalization and low side effects of encapsulated drugs, while keeping therapeutic efficacy. It is interesting to note that within the tumour tissue (acidic $\mathrm{pH}$ ) the system exhibited negative to positive charge reversal, facilitating the cell internalization and subsequent nuclear targeting. The anticancer drug model used, 10-hydroxycamptothecin, was conjugated to methoxy polyethylene glycol to improve the cytotoxicity.

Liposomes are perhaps the most successful formulation for clinical drug delivery. Since they will be discussed in detail in the next Section regarding their in vivo application, here we will only mention that surface-modified liposomes having arginine-rich cell penetrating peptides and transferrin [67] were reported to improve targeting onto A2780 ovarian carcinoma cells via the over-expressed transferrin receptors. In the same way Patil et al. [68] reported liposomes containing a dual chemotherapeutic load of lipophilic mitomycin $\mathrm{C}$ and doxorubicin.

A lipid-polymer hybrid liposomal nano-platform based on hyaluronic acid-magnetic nanoparticle-liposomes have shown also good results as a vehicle for docetaxel by improving the cellular uptake in human breast cancer cells.[69] In addition, they proved the double effect of chemotherapy and thermotherapy under near-infrared laser irradiation (NIR, 808 $\mathrm{nm}) .[70]$

As mentioned in Section 1, inorganic metallic NPs have also been exploited to transport and release therapeutic drugs by chemical or physical stimuli. The most extensively investigated material is Au-NPs. Functionalized gold nanoparticles are known to have good biocompatibility and versatile shape and size, providing a tunable response to physical stimuli. Recently, Hernández Montoro et al. [71] tested a drug release system based on Au-nanostars coated with a mesoporous silica in HeLa cells. The silica shell was loaded with doxorubicin (Dox) and coated with octadecyltrimethoxy-silane and paraffin to retard the release of Dox. Low cytotoxicity confirmed the efficacy of the coating to prevent Dox leaking, and using a $808 \mathrm{~nm}$ laser irradiation the release profile was accelerated. Spherical Au-NPs have been also tested in vitro, using different coatings, with different degrees of success.[72]

The development of drug-vehicles made of materials with dual effect has also witnessed some successful in vitro experimentation. Rodrigues et al. [73] used graphene-based magnetic nanoparticles functionalized with pluronic F-127, demonstrating efficacy on concurrent hyperthermia and $\mathrm{pH}$ stimuli-responsive drug delivery on Hep-G2 cells. This nanosystem showed the potential ability to transport therapeutic doses of doxorubicin, and to respond to both $\mathrm{pH}$ changes in tumour environment and to external magnetic fields. In addition, albumin-based MNPs with low toxicity and immunogenicity, have been tested in vitro by Nostari et al. [74], who applied albumin-coated MNPs attached with curcumin (CUR) in vitro, aiming to decrease tumour growth through proliferation suppression. Although this compound presents really promising properties, it is not commonly used due to the short biological half-life and low solubility, which results in poor absorption and thus low bioavailability through the oral route.

\section{TARGETED NANOCARRIERS FOR IN VIVO DRUG DELIVERY}

Current nano-based drug delivery systems (DDS) are still far from the concept of "magic bullet" created by Paul Ehrlich in early 1900's. Most of them rely on passive drug accumulation in desired tissues, taking advantage of physiological conditions like the EPR effect, which has been described since approximately 30 years.[75] However, only a marginal increase in drug concentration has been reached with passively guided strategies, and this often represents only a very small fraction of the total dose administered. For this reason, active targeting is necessary to achieve specific tissue/organ accumulation. Active drug transport is based on molecular recognition processes to deliver drugs to specific pathological sites. Most active guided nanosystems employ targeting ligands at their surface, including organic molecules, antibodies, aptamers, proteins or peptides.[76]

Drug delivery to the central nervous system (CNS) is often limited by the very low permeability of the blood-brain barrier (BBB), which is composed of a continuous and chargepolarized endothelial cell layer. Therefore, most of the drugs must travel across a trans-cellular hurdle employing a set of transporters before targeting brain tissues. There are already good recent reviews on the use of diverse nanocarriers for treating neurodegenerative and other CNS diseases [77, 78] and thus here we mention only some examples. For instance, a PEGylated liposomal system functionalized with transferrin (Tf) and penetratin (Pen) for targeting BBB endothelial cells was recently tested for transfection both in vitro and in vivo.[79] In the latter, this PenTf-liposome was used to assess gene delivery of $\beta$-galactosidase-peptide through monitoring $\beta$-galactosidase expression in mice tissues. The results evidenced that PenTf-liposomes were able to cross BBB and 
accumulate in brain (12\% of the injected dose), and had better transfection capacity than plain liposomes.

Recently, Shahin et al. [80] synthesized a nanocarrier based on PEI-coated mesoporous silica nanoparticles (MSNs) conjugated with hyaluronic acid (HA) for targeting CD44 expressing-recurrent ovarian cancer stem cells. A novel small interfering RNA against TWIST protein (siTWIST) was further conjugated with the MSNs-HA particles aiming to suppress TWIST and reducing chemoresistance when administered in combination with cisplatin. In vivo results showed 60\% tumour growth suppression in the cisplatin group compared to control, MSN-siTWIST reduced it an additional 20\%, but the MSN-HA-siTWIST + cisplatin group exhibited the best efficacy, reducing almost a $90 \%$ the tumor volume. Future work could show the potential applications of this system in drug delivery.

Another option for active drug delivery of antineoplastic drugs is targeting the tumour vasculature. RGD peptide can bind to $\alpha_{v} \beta 5$ integrin, which is often overexpressed in some types of tumours. Song et al. [81] developed a novel nanostructured lipid carrier composed of a mixture of solid and liquid state lipids conjugated with RGD and encapsulating anticancer drug temozolomide (TMZ) for glioblastoma multiforme treatment. RGD-TMZ/NLCs efficacy were tested on U87 MG-bearing mice, treatments were given intravenously and repeated once every three days over a period of 21 days. At the end of the study, RGD-TMZ/NLCs showed a 4-fold inhibition of tumour growth compared with the free drug treatment group. Furthermore, tumour growth inhibition was significantly increased compared with nonRGD guided NLCs, confirming active targeting to tumour vasculature via RGD peptide.

\subsection{Stimuli-responsive control of drug release}

Controlling drug release rates using external stimuli on nanocarriers at target sites is a persistent challenge. This noninvasive strategy has still many issues that need to be solved before it can be safely translated into clinics. The research of new components for nanocarriers has allowed the development of systems with dual or multi stimuli-triggered drug release mechanisms through both internal $(\mathrm{pH}$, temperature, redox potential, ROS generation, enzymes, hypoxia) and external (ultrasound, light, radiofrequencies, magnetic fields) stimulus. There are many tests currently running on these novel carriers, both in vitro and in vivo. [82],[83],[84]

The liposomal formulations are perhaps the more successful DDS currently employed in clinics, due to their physicochemical compatibility, pharmacokinetic behaviour and delivery efficacy. Thus, liposomes have been used as the starting point to build many stimuli-responsive DDS. For example, pegylated liposomes loaded with cisplatin and MNPs (i.e., magnetoliposomes) have been tested in vitro and in vivo regarding their transition temperature (Tm), encapsulation efficiency and drug release profiles.[85]. This new type of DDS showed the capacity of inhibiting tumour growth, and when combined with magnetic hyperthermia treatments a complete obliteration of the tumours was reported.[85]
Another promising formulation of magneto-liposomes encapsulating both iron oxide magnetic nanoparticles and a commercially available photosensitizer (Foscan, m-THPC) was tested for a combination of magnetic hyperthermia (MHT) and photodynamic therapy (PDT) for tumour ablation. The formulation yielded satisfactory ratios of both agents and showed good stability in vitro (less than $20 \%$ of m-THPC released during the first seven days). In vivo, combination of MHT and PDT with the m-THPC-loaded MLPs showed better efficacy than individual treatments and was able to completely eradicate tumours in A431 xenografts.[86]

After the first applications of hyperthermia, surviving cancer cells often develop thermo-resistance associated with increased expression of heat shock proteins (HSPs) reducing subsequent heating efficacy and tumour relapses. 17-AGG, an HSP90 inhibitor derived from geldanamycin, has shown capacity for killing tumour cells by inhibiting HSP90, however, its poor solubility in water limits its use. In order to overcome this limitation, Yang et al. [87] encapsulated 17AGG inside thermosensitive MLPs and further modified them for targeting $\mathrm{CD} 0^{+}$liver cancer stem cells (CD90@17AGG/TMs). To prove the antitumor efficacy of the formulation, CD90@17-AGG/TMs were intratumorally injected in CD $90^{+} \mathrm{LCSC}$-bearing mice and $24 \mathrm{~h}$ after administration, tumours were exposed to an alternating current magnetic field (ACMF, $\mathrm{f}=200 \mathrm{kHz}, \mathrm{I}=20 \mathrm{~A}$ ) for $1 \mathrm{~h}$, every other day for a week. Compared to the other experimental groups, CD90@17-AGG/TMs + ACFM showed superior reduction of tumour size $(83.44 \pm 5.78 \%$ inhibition rate) and increased number of apoptotic cells in tumour tissue. Dual stimuli-responsive polymeric micelles composed of methoxy poly(ethylene glycol)-co-poly (lysine) (mPEGPLys) and a mixture of fatty acids including linoleic acid, $\alpha$ linoleic acid and araquidonic acid as building blocks were developed by Gao et al. Chlorin e6 (Ce6) was loaded within the micelle core. Ce6 is a photosensitizer, which, upon laser irradiation, can produce highly reactive oxygen species (ROS). These ROS not only exerts a cytotoxic effect on cancer cells but also act like a trigger mechanism, causing lipid peroxidation of the unsaturated fatty acids and destabilizing the vehicle for a rapid release of $\mathrm{Ce} 6$ and a maximization of the ROS effects. This hypothesis was tested in vivo in tumourbearing mice after intravenous administration of either free $\mathrm{Ce} 6$ or its micellar form $\left(\mathrm{RM}_{\mathrm{AA}}\right)$. The authors found that after $24 \mathrm{~h}$ post administration, Ce6 concentration in tumour tissue was much higher in $\mathrm{RM}_{\mathrm{AA}}$ group; also $\mathrm{RM}_{\mathrm{AA}}$ exhibited the best antitumor outcome reducing tumour volume and improving survival time[88].

Shi et al. demonstrated another example of ROS-mediated drug release.[89] These authors succeeded in formulating mesoporous $\mathrm{TiO}_{2}$ nanoparticles with entrapped doxorubicin and coupling dsDNA fragments at the surface to block doxorubicin leakage. The nanosystem exhibited very good stability under physiological conditions. However, under ultrasound stimulation $\mathrm{TiO}_{2}$ can produce ROS that causes dsDNA shearing and therefore increasing doxorubicin release. This was first demonstrated in vitro, where nanoparticles without ultrasound stimulation released only $11 \%$ of encapsulated doxorubicin after $48 \mathrm{~h}$ while $83 \%$ was released when ultrasound was applied. These results 
encouraged authors to analyse the possible antitumor efficacy in vivo. NBMTNs were intravenously injected in MCF7/ADR tumour bearing mice and ultrasound irradiation $\left(1 \mathrm{~W} / \mathrm{cm}^{2}, 120 \mathrm{~S}\right)$ was performed for $24 \mathrm{~h}$ on tumour site. Results showed that NBMTNs + US causes a 5-fold higher inhibition of tumour growth compared with control group and 4-fold inhibition compared with free doxorubicin or NBMTNs without ultrasound.

A novel system composed by redox/enzyme self-assembling polymeric NPs has shown promising results.[90] This complex system was obtained by conjugating chondroitin sulfate (CS) with deoxicholic acid (DOCA) through a redoxsensitive disulfide bond. A therapeutic drug, docetaxel (DTX), was further encapsulated within these nanoparticles. Hyal-1 is a lysosomal enzyme overexpressed in various types of cancer cells, associated with growth, metastasis and angiogenesis of tumours. Since HA can be degraded by Hyal1 and the structural similarities between CS and HA, it is believed that Hyal-1 can also degrade CS. Therefore, controlled release in these nanoparticles can be achieved by Hyal-1 induced CS degradation and glutation (GSH) induced cleavage of disulphide bonds. This hypothesis was tested both in vitro in $\mathrm{B} 16 \mathrm{~F} 10$ cells (murine melanoma) and in vivo using B16F10 tumour bearing mice. Compared with commercial docetaxel (Taxotere $\left.{ }^{\circledR}\right)$, CSCD showed a 4-fold higher area under the receiver-operating curve in tumor. Furthermore, CSCD showed increased tumor growth inhibition and reduced lung metastasis.

Theranostic nanosystems appear to be the next step towards personalized medicine. Formulations that can be applied for both diagnostic and therapeutic purpose have attracted much attention in the last years. As an example, the recently reported nanosystem made of liposomes with co-encapsulated perfluorocarbon (PFC), hollow gold nanospheres (HAuNS), and doxorubicin (DOX), was intended for ultrasound imaging, photothermal therapy (PTT) and temperaturecontrolled drug release purposes, all triggered by NIR light irradiation. This and other types of new, multipurpose nanosystems have started to provide quite promising results both in vitro and in vivo.[91]

\subsection{Translation into clinics}

Nanotechnology came into the field of drug delivery systems (DDS) about two decades ago, opening new ways to improve the spatial and temporal control of the therapeutic release profiles. This resulted in a large number of new concepts and nanodevices with proven experimental success in vitro or in vivo. But only a much smaller fraction of all these new DDS developed was able to obtain approval to be used in human clinics.

Figure 3 resumes the main key-points involved from the initial development of any DDS to the final clinical approval and commercialization. In spite of the fact that many new developments subjected to clinical trials use clinically approved drugs, a full characterization phase for each DDS is required since small changes in composition or physicochemical parameters like particle size, zeta potential or drug-release patterns could cause a completely different behaviour. After the formulation phase, preclinical studies both in vitro and in vivo are necessary to prove the bioavailability, efficacy and safety properties of the nanocarrier before their use in patients. Furthermore, clinical studies from phase I to phase III must guarantee the bioequivalence, safety profile and better/similar efficacy compared with the standard drug before finally being approved in clinics.

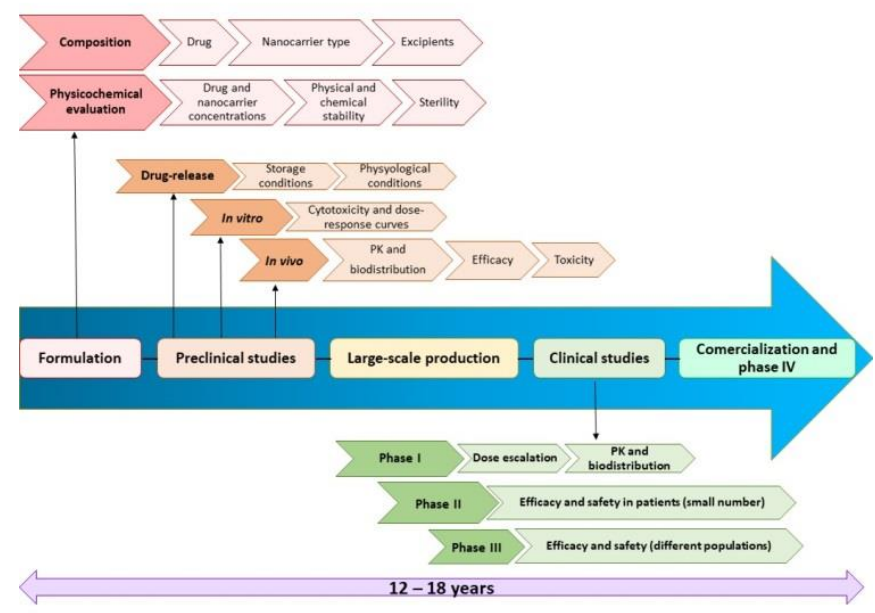

Figure 3 Key-points along the efficacy/safety studies for new nanomaterialbased drug delivery systems, before they can be approved for clinical uses.

In the last few years, a great number of experimental nanocarriers have been developed and tested in preclinical stages.[92] Unfortunately, despite the substantial research showing promising results both in vitro and in vivo with this nanomaterials for controlled drug delivery and release, there are only few of them that have been successfully translated to clinical studies and even less have been approved for clinical use (see Table 1). However, owing to the increasing information in molecular biology of different diseases and the positive results in preclinical stages, the number of nanomaterials in clinical stages is expected to increase in the next years.[93],[94],[95]

\section{CONCLUSION}

Many long-standing glitches that hindered therapeutic applications of drug delivery systems (DDS) have been overcome by the incorporation of nanotechnology and nanomaterials into the main original conception. Although solid matrix formulations are still irreplaceable for some therapeutic goals, nanosystems with active control of release kinetics are now a real possibility. The review of the current literature shows that those DDS currently available have improved the pharmacological outcomes of several therapeutic strategies, making a real impact on the clinical activity. Among the DDS formulations with evident success, anticancer-drug delivery systems stand out. Moreover, the basic templates already developed for some clinicallyapproved DDS can be adapted for new drugs still to be synthesized from the (also) fast-growing field of chemical engineering and drug screening. On the other hand, the success regarding DDS having remote wireless control of drug release is yet to be proven at clinical stages. These would 
be the next generation DDS that could resemble the longstanding conception of a 'magic bullet'.

\section{LIST OF ABBREVIATIONS}

DDS: drug delivery systems; EVs: Extracellular vesicles; EMF: electromagnetic field; RF: radiofrequency; NPs: Nanoparticles; MNPs: Magnetic nanoparticles; MHT: Magnetic Hyperthermia; Dox: Doxorubicin; PFC: perfluorocarbon; HAuNS: hollow gold nanospheres; MLP: magneto-liposome; PPT: photothermal therapy; ROS: reactive oxygen species; TMZ: temozolomide; CNS: central nervous system; BBB: blood-brain barrier.

\section{CONFLICT OF INTEREST}

The authors declare that there is no conflict of interest.

\section{ACKNOWLEDGEMENTS}

The authors acknowledge financial support from the Spanish Ministerio de Ciencia, Innovación y Universidades (project MAT2016-78201-P) and the Aragon Regional Government (DGA, Project No. E26).

\section{REFERENCES}

[1] Nystrom C, Bisrat M. PHYSICOCHEMICAL ASPECTS OF DRUG RELEASE .3. COULTER-COUNTER MEASUREMENTS OF SOLUBILITY AND DISSOLUTION RATE OF SPARINGLY SOLUBLE COMPOUNDS USING MICELLAR SOLUTIONS. J Pharm Pharmacol. 1986;38:420-5.

[2] Bazile D, Prudhomme C, Bassoullet MT, Marlard M, Spenlehauer G, Veillard M. STEALTH ME.PEG-PLA NANOPARTICLES AVOID UPTAKE BY THE MONONUCLEAR PHAGOCYTES SYSTEM. J Pharm Sci. 1995;84:493-8.

[3] Bangham AD, Horne R. Negative staining of phospholipids and their structural modification by surfaceactive agents as observed in the electron microscope. Journal of molecular biology. 1964;8:660-IN10.

[4] Mezei M, Gulasekharam V. Liposomes-a selective drug delivery system for the topical route of administration I. Lotion dosage form. Life Sciences. 1980;26:1473-7.

[5] Sarangi B, Jana U, Mohanta GP, Manna PK. Drug Release Kinetics Study of Lovastatin Loaded Solid Lipid Nanoparticles for Oral Delivery. Current Nanoscience. 2018;14:319-28.

[6] Soussan E, Cassel S, Blanzat M, Rico-Lattes I. Drug Delivery by Soft Matter: Matrix and Vesicular Carriers. Angewandte Chemie International Edition. 2009;48:274-88.

[7] Armstrong JPK, Stevens MM. Strategic design of extracellular vesicle drug delivery systems. Adv Drug Deliv Rev. 2018;130:12-6.

[8] Sun D, Zhuang X, Xiang X, Liu Y, Zhang S, Liu C, et al. A Novel Nanoparticle Drug Delivery System: The Antiinflammatory Activity of Curcumin Is Enhanced When Encapsulated in Exosomes. Molecular Therapy. 2010;18:1606-14.
[9] El Andaloussi S, Maeger I, Breakefield XO, Wood MJA. Extracellular vesicles: biology and emerging therapeutic opportunities. Nat Rev Drug Discov. 2013;12:348-58.

[10] Yang T, Martin P, Fogarty B, Brown A, Schurman K, Phipps R, et al. Exosome Delivered Anticancer Drugs Across the Blood-Brain Barrier for Brain Cancer Therapy in Danio Rerio. Pharm Res. 2015;32:2003-14.

[11] Stoorvogel W, Kleijmeer MJ, Geuze HJ, Raposo G. The biogenesis and functions of exosomes. Traffic. 2002;3:32130.

[12] El-Boubbou K. Magnetic iron oxide nanoparticles as drug carriers: Preparation, conjugation and delivery. Nanomedicine. 2018;13:929-52.

[13] Giannaccini M, Calatayud MP, Poggetti A, Corbianco S, Novelli M, Paoli M, et al. Magnetic Nanoparticles for Efficient Delivery of Growth Factors: Stimulation of Peripheral Nerve Regeneration. Advanced healthcare materials. 2017;6.

[14] Cao Y, Chen Y, Yu T, Guo Y, Liu F, Yao Y, et al. Drug Release from Phase-Changeable Nanodroplets Triggered by Low-Intensity Focused Ultrasound. Theranostics. 2018;8:1327-39.

[15] Schroeder A, Avnir Y, Weisman S, Najajreh Y, Gabizon A, Talmon Y, et al. Controlling liposomal drug release with low frequency ultrasound: mechanism and feasibility. Langmuir : the ACS journal of surfaces and colloids. 2007;23:4019-25.

[16] Schwerdt JI, Goya GF, Calatayud MP, Herenu CB, Reggiani PC, Goya RG. Magnetic field-assisted gene delivery: achievements and therapeutic potential. Current gene therapy. 2012;12:116-26.

[17] McNamara K, Tofail SAM. Nanoparticles in biomedical applications. Advances in Physics: X. 2017;2:54-88.

[18] Noh YW, Lim YT, Chung BH. Noninvasive imaging of dendritic cell migration into lymph nodes using near-infrared fluorescent semiconductor nanocrystals. FASEB journal : official publication of the Federation of American Societies for Experimental Biology. 2008;22:3908-18.

[19] Huang L, Wan J, Wei X, Liu Y, Huang J, Sun X, et al. Plasmonic silver nanoshells for drug and metabolite detection. Nature communications. 2017;8:220.

[20] Espana-Sanchez BL, Avila-Orta CA, Padilla-Vaca LF, Barriga-Castro ED, Soriano-Corral F, Gonzalez-Morones P, et al. Early Stages of Antibacterial Damage of Metallic Nanoparticles by TEM and STEM-HAADF. Current Nanoscience. 2018;14:54-61.

[21] Dykman LA, Khlebtsov NG. Multifunctional gold-based nanocomposites for theranostics. Biomaterials. 2016;108:1334.

[22] Panchapakesan B, Book-Newell B, Sethu P, Rao M, Irudayaraj J. Gold nanoprobes for theranostics. Nanomedicine (Lond). 2011;6:1787-811.

[23] Koyani R, Perez-Robles J, Cadena-Nava RD, VazquezDuhalt R. Biomaterial-based nanoreactors, an alternative for enzyme delivery. Nanotechnol Rev. 2017;6:405-19.

[24] Torchi A, Simonelli F, Ferrando R, Rossi G. Local Enhancement of Lipid Membrane Permeability Induced by Irradiated Gold Nanoparticles. ACS nano. 2017;11:12553-61. 
[25] Jiang B, Li C, Dag O, Abe H, Takei T, Imai T, et al. Mesoporous metallic rhodium nanoparticles. Nature communications. 2017;8.

[26] Figge FH, Flower Jr G. Method of administering therapeutic agents. In: Inc A, editor. USA: AMP Inc; 1969.

[27] Thanh NT. Clinical Applications of Magnetic Nanoparticles: From Fabrication to Clinical Applications: CRC Press; 2018.

[28] Dobson J, El Haj AJ, Bin H, Markides H, Henstock JR. Applications of Magnetic Nanoparticles in Tissue Engineering and Regenerative Medicine. Nanomagnetic Actuation in Biomedicine: CRC Press; 2018. p. 205-28.

[29] Chiu-Lam A, Rinaldi C. Nanoscale thermal phenomena in the vicinity of magnetic nanoparticles in alternating magnetic fields. Advanced functional materials. 2016;26:3933-41.

[30] Alphandery E, Haidar DA, Seksek O, Thoreau M, Trautmann A, Bercovici N, et al. A Fluorescent Nanoprobe for the Detection of in Situ Temperature Changes during Hyperthermia Treatment of Tumors. Biophysical Journal. 2018;114:361a.

[31] Hayashi K, Ono K, Suzuki H, Sawada M, Moriya M, Sakamoto W, et al. High-Frequency, Magnetic-FieldResponsive Drug Release from Magnetic Nanoparticle/Organic Hybrid Based on Hyperthermic Effect. ACS Appl Mater Interfaces. 2010;2:1903-11.

[32] Hernández R, Sacristán J, Asín L, Torres TE, Ibarra MR, Goya GF, et al. Magnetic Hydrogels Derived from Polysaccharides with Improved Specific Power Absorption: Potential Devices for Remotely Triggered Drug Delivery. The Journal of Physical Chemistry B. 2010;114:12002-7.

[33] Criado M, Sanz B, Goya GF, Mijangos C, Hernández R. Magnetically responsive biopolymeric multilayer films for local hyperthermia. Journal of Materials Chemistry B. 2017;5:8570-8.

[34] Guo Y, Zhang Y, Ma J, Li Q, Li Y, Zhou X, et al. Light/magnetic hyperthermia triggered drug released from multi-functional thermo-sensitive magnetoliposomes for precise cancer synergetic theranostics. J Control Release. 2018;272:145-58.

[35] Tietze R, Zaloga J, Unterweger H, Lyer S, Friedrich RP, Janko C, et al. Magnetic nanoparticle-based drug delivery for cancer therapy. Biochem Biophys Res Commun. 2015;468:463-70.

[36] Paixão P, Gouveia LF, Silva N, Morais JAG. Evaluation of dissolution profile similarity - Comparison between the $\mathrm{f} 2$, the multivariate statistical distance and the f2 bootstrapping methods. European Journal of Pharmaceutics and Biopharmaceutics. 2017;112:67-74.

[37] Bibby DC, Davies NM, Tucker IG. Mechanisms by which cyclodextrins modify drug release from polymeric drug delivery systems. Int J Pharm. 2000;197:1-11.

[38] Chiou WL, Riegelman S. Pharmaceutical applications of solid dispersion systems. J Pharm Sci. 1971;60:1281-302.

[39] Juarez JM, Cussa J, Gomez Costa MB, Anunziata OA. Nanostructured Ketorolac-Tromethamine/MCF: Synthesis, Characterization and Application in Drug Release System. Current Nanoscience. 2018;14:432-9.
[40] Corrigan OI. Mechanisms of dissolution of fast release solid dispersions. Drug development and industrial pharmacy. 1985;11:697-724.

[41] Craig DQ. The mechanisms of drug release from solid dispersions in water-soluble polymers. Int $\mathrm{J}$ Pharm. 2002;231:131-44.

[42] Quaglia F, Varricchio G, Miro A, La Rotonda MI, Larobina D, Mensitieri G. Modulation of drug release from hydrogels by using cyclodextrins: the case of nicardipine/beta-cyclodextrin system in crosslinked polyethylenglycol. J Control Release. 2001;71:329-37.

[43] Aloisio C, de Oliveira AG, Longhi M. Characterization, inclusion mode, phase-solubility and in vitro release studies of inclusion binary complexes with cyclodextrins and meglumine using sulfamerazine as model drug. Drug development and industrial pharmacy. 2014;40:919-28.

[44] Sastry SV, Nyshadham JR, Fix JA. Recent technological advances in oral drug delivery-a review. Pharmaceutical science \& technology today. 2000;3:138-45.

[45] Lee SS, Lim CB, Pai CM, Lee S, Park I, Seo MG, et al. Composition and pharmaceutical dosage form for colonic drug delivery using polysaccharides. Google Patents; 2002.

[46] Wang Y, Kohane DS. External triggering and triggered targeting strategies for drug delivery. Nature Reviews Materials. 2017;2:17020.

[47] Meers P. Enzyme-activated targeting of liposomes. Adv Drug Deliv Rev. 2001;53:265-72.

[48] Burke C, Dreher MR, Negussie AH, Mikhail AS, Yarmolenko P, Patel A, et al. Drug release kinetics of temperature sensitive liposomes measured at high-temporal resolution with a millifluidic device. International Journal of Hyperthermia. 2018;34:786-94.

[49] Vankayala R, Hwang KC. Near-Infrared-LightActivatable Nanomaterial-Mediated Phototheranostic Nanomedicines: An Emerging Paradigm for Cancer Treatment. Adv Mater. 2018;30:1706320.

[50] Hoare T, Timko BP, Santamaria J, Goya GF, Irusta S, Lau S, et al. Magnetically triggered nanocomposite membranes: a versatile platform for triggered drug release. Nano letters. 2011;11:1395-400.

[51] Salkho N, Turki R, Guessoum O, Martins A, Vitor R, Husseini G. Liposomes as a Promising Ultrasound-Triggered Drug Delivery System in Cancer Treatment. Current molecular medicine. 2017;17:668-88.

[52] Soltys M, Kovatcik P, Lhotka M, Ulbrich P, Zadrazil A, Stepanek F. Radiofrequency controlled release from mesoporous silica nano-carriers. Microporous Mesoporous Mat. 2016;229:14-21.

[53] Cezar CA, Kennedy SM, Mehta M, Weaver JC, Gu L, Vandenburgh H, et al. Biphasic Ferrogels for Triggered Drug and Cell Delivery. Advanced healthcare materials. 2014;3:1869-76.

[54] Pradhan P, Giri J, Rieken F, Koch C, Mykhaylyk O, Doblinger $\mathrm{M}$, et al. Targeted temperature sensitive magnetic liposomes for thermo-chemotherapy. J Control Release. 2010;142:108-21. 
[55] Budhwani KI, Dettmann MA, Saleh MN, Thomas V. Nano and Microbubble Systems for On-Demand Cancer Drug Delivery. Current Nanoscience. 2018;14:33-41.

[56] Huang SL, Hamilton AJ, Nagaraj A, Tiukinhoy SD, Klegerman ME, Mcpherson DD, et al. Improving ultrasound reflectivity and stability of echogenic liposomal dispersions for use as targeted ultrasound contrast agents. J Pharm Sci. 2001;90:1917-26.

[57] Centelles MN, Wright M, So P-W, Amrahli M, Xu XY, Stebbing J, et al. Image-guided thermosensitive liposomes for focused ultrasound drug delivery: Using NIRF-labelled lipids and topotecan to visualise the effects of hyperthermia in tumours. J Control Release. 2018;280:87-98.

[58] Fite BZ, Kheirolomoom A, Foiret JL, Seo JW, Mahakian LM, Ingham ES, et al. Dynamic contrast enhanced MRI detects changes in vascular transport rate constants following treatment with thermally-sensitive liposomal doxorubicin. J Control Release. 2017;256:203-13.

[59] Klibanov AL. Ligand-carrying gas-filled microbubbles: ultrasound contrast agents for targeted molecular imaging. Bioconjug Chem. 2005;16:9-17.

[60] Salomon J, Doelker E. Formulation des comprimes à liberation prolongee I. Matrices inertes. Pharm Acta Helv. 1980;55:174-82.

[61] Mukherjee S, Shunmugam R. Polymer based NanoAssemblies: Very Efficient Carrier in the Field of Cancer Chemotherapy. J Nanomed Res. 2017;5:00137.

[62] Olden BR, Cheng Y, Yu JL, Pun SH. Cationic polymers for non-viral gene delivery to human $\mathrm{T}$ cells. Journal of controlled release : official journal of the Controlled Release Society. 2018;282:140-7.

[63] Khoshnejad M, Parhiz H, Shuvaev VV, Dmochowski IJ, Muzykantov VR. Ferritin-based drug delivery systems: Hybrid nanocarriers for vascular immunotargeting. Journal of controlled release : official journal of the Controlled Release Society. 2018;282:13-24.

[64] Zhang L, Li L, Di Penta A, Carmona U, Yang F, Schöps R, et al. H-Chain Ferritin: A Natural Nuclei Targeting and Bioactive Delivery Nanovector. Advanced healthcare materials. 2015;4:1305-10.

[65] Kim S, Kim GS, Seo J, Gowri Rangaswamy G, So IS, Park RW, et al. Double-Chambered Ferritin Platform: DualFunction Payloads of Cytotoxic Peptides and Fluorescent Protein. Biomacromolecules. 2016;17:12-9.

[66] Jing Y, Xiong X, Ming Y, Zhao J, Guo X, Yang G, et al. A Multifunctional Micellar Nanoplatform with $\mathrm{pH}$-Triggered Cell Penetration and Nuclear Targeting for Effective Cancer Therapy and Inhibition to Lung Metastasis. Advanced healthcare materials. 2018;7:1700974.

[67] Deshpande P, Jhaveri A, Pattni B, Biswas S, Torchilin V. Transferrin and octaarginine modified dual-functional liposomes with improved cancer cell targeting and enhanced intracellular delivery for the treatment of ovarian cancer. Drug delivery. 2018;25:517-32.

[68] Patil Y, Shmeeda H, Amitay Y, Ohana P, Kumar S, Gabizon A. Targeting of folate-conjugated liposomes with coentrapped drugs to prostate cancer cells via prostate-specific membrane antigen (PSMA). Nanomedicine: Nanotechnology, Biology and Medicine. 2018;14:1407-16.

[69] Nguyen VD, Zheng S, Han J, Le VH, Park JO, Park S. Nanohybrid magnetic liposome functionalized with hyaluronic acid for enhanced cellular uptake and nearinfrared-triggered drug release. Colloids and surfaces B, Biointerfaces. 2017;154:104-14.

[70] Hardiansyah A, Yang M-C, Liu T-Y, Kuo C-Y, Huang L-Y, Chan T-Y. Hydrophobic drug-loaded PEGylated magnetic liposomes for drug-controlled release. Nanoscale research letters. 2017;12:355.

[71] Hernández Montoto A, Montes R, Samadi A, Gorbe M, Terrés JM, Cao-Milán R, et al. Gold Nanostars Coated with Mesoporous Silica Are Effective and Nontoxic Photothermal Agents Capable of Gate Keeping and Laser-Induced Drug Release. ACS Appl Mater Interfaces. 2018;10:27644-56.

[72] Khutale GV, Casey A. Synthesis and characterization of a multifunctional gold-doxorubicin nanoparticle system for $\mathrm{pH}$ triggered intracellular anticancer drug release. European Journal of Pharmaceutics and Biopharmaceutics. 2017; 119:372-80.

[73] Rodrigues RO, Baldi G, Doumett S, Garcia-Hevia L, Gallo J, Bañobre-López M, et al. Multifunctional graphenebased magnetic nanocarriers for combined hyperthermia and dual stimuli-responsive drug delivery. Materials Science and Engineering: C. 2018;93:206-17.

[74] Nosrati H, Sefidi N, Sharafi A, Danafar H, Manjili HK. Bovine serum albumin (BSA) coated iron oxide magnetic nanoparticles as biocompatible carriers for curcuminanticancer drug. Bioorganic chemistry. 2018;76:501-9.

[75] Maeda H, Nakamura H, Fang J. The EPR effect for macromolecular drug delivery to solid tumors: Improvement of tumor uptake, lowering of systemic toxicity, and distinct tumor imaging in vivo. Adv Drug Deliv Rev. 2013;65:71-9.

[76] Kumari P, Ghosh B, Biswas S. Nanocarriers for cancertargeted drug delivery. Journal of drug targeting. 2016;24:179-91.

[77] Karthivashan G, Ganesan P, Park SY, Kim JS, Choi DK. Therapeutic strategies and nano-drug delivery applications in management of ageing Alzheimer's disease. Drug Deliv. 2018;25:307-20.

[78] Saraiva C, Praca C, Ferreira R, Santos T, Ferreira L, Bernardino L. Nanoparticle-mediated brain drug delivery: Overcoming blood-brain barrier to treat neurodegenerative diseases. Journal of controlled release : official journal of the Controlled Release Society. 2016;235:34-47.

[79] Dos Santos Rodrigues B, Oue H, Banerjee A, Kanekiyo T, Singh J. Dual functionalized liposome-mediated gene delivery across triple co-culture blood brain barrier model and specific in vivo neuronal transfection. Journal of controlled release : official journal of the Controlled Release Society. 2018;286:264-78.

[80] Shahin SA, Wang R, Simargi SI, Contreras A, Parra Echavarria L, Qu L, et al. Hyaluronic acid conjugated nanoparticle delivery of siRNA against TWIST reduces tumor burden and enhances sensitivity to cisplatin in ovarian cancer. Nanomedicine. 2018;14:1381-94. 
[81] Song S, Mao G, Du J, Zhu X. Novel RGD containing, temozolomide-loading nanostructured lipid carriers for glioblastoma multiforme chemotherapy. Drug Deliv. 2016;23:1404-8.

[82] Kalaydina RV, Bajwa K, Qorri B, Decarlo A, Szewczuk MR. Recent advances in "smart" delivery systems for extended drug release in cancer therapy. International journal of nanomedicine. 2018;13:4727-45.

[83] Lim EK, Chung BH, Chung SJ. Recent Advances in pHSensitive Polymeric Nanoparticles for Smart Drug Delivery in Cancer Therapy. Current drug targets. 2018;19:300-17.

[84] Mai BT, Fernandes S, Balakrishnan PB, Pellegrino T. Nanosystems Based on Magnetic Nanoparticles and Thermoor pH-Responsive Polymers: An Update and Future Perspectives. Accounts of chemical research. 2018;51:9991013.

[85] Toro-Cordova A, Flores-Cruz M, Santoyo-Salazar J, Carrillo-Nava E, Jurado R, Figueroa-Rodriguez P, et al. Liposomes Loaded with Cisplatin and Magnetic Nanoparticles: Physicochemical Characterization, Pharmacokinetics, and In-Vitro Efficacy. Molecules. 2018;23:2272.

[86] Di Corato R, Bealle G, Kolosnjaj-Tabi J, Espinosa A, Clement O, Silva AK, et al. Combining magnetic hyperthermia and photodynamic therapy for tumor ablation with photoresponsive magnetic liposomes. ACS nano. 2015;9:2904-16.

[87] Yang R, Tang Q, Miao F, An Y, Li M, Han Y, et al. Inhibition of heat-shock protein 90 sensitizes liver cancer stem-like cells to magnetic hyperthermia and enhances antitumor effect on hepatocellular carcinoma-burdened nude mice. International journal of nanomedicine. 2015;10:734558.

[88] Gao M, Meng X, Guo X, Zhu J, Fan A, Wang Z, et al. All-active antitumor micelles via triggered lipid peroxidation.
Journal of controlled release : official journal of the Controlled Release Society. 2018;286:381-93.

[89] Shi J, Liu W, Fu Y, Yin N, Zhang H, Chang J, et al. "USdetonated nano bombs" facilitate targeting treatment of resistant breast cancer. Journal of controlled release : official journal of the Controlled Release Society. 2018;274:9-23.

[90] Liu M, Du H, Khan AR, Ji J, Yu A, Zhai G. Redox/enzyme sensitive chondroitin sulfate-based selfassembled nanoparticles loading docetaxel for the inhibition of metastasis and growth of melanoma. Carbohydrate polymers. 2018;184:82-93.

[91] Li W, Hou W, Guo X, Luo L, Li Q, Zhu C, et al. Temperature-controlled, phase-transition ultrasound imagingguided photothermal-chemotherapy triggered by NIR light. Theranostics. 2018;8:3059-73.

[92] Marchal S, El Hor A, Millard M, Gillon V, Bezdetnaya L. Anticancer Drug Delivery: An Update on Clinically Applied Nanotherapeutics. Drugs. 2015;75:1601-11.

[93] Park K. Controlled drug delivery systems: past forward and future back. Journal of controlled release : official journal of the Controlled Release Society. 2014;190:3-8.

[94] Ulbrich K, Hola K, Subr V, Bakandritsos A, Tucek J, Zboril R. Targeted Drug Delivery with Polymers and Magnetic Nanoparticles: Covalent and Noncovalent Approaches, Release Control, and Clinical Studies. Chemical reviews. 2016;116:5338-431.

[95] Kralj S, Potrc T, Kocbek P, Marchesan S, Makovec D. Design and Fabrication of Magnetically Responsive Nanocarriers for Drug Delivery. Current medicinal chemistry. 2017;24:454-69. 
Table 1. Nanotechnology-based drug delivery systems approved for clinical use

\begin{tabular}{|c|c|c|c|}
\hline Category & Name & Drug & Disease \\
\hline Nanocrystals & ZYPAdhera & Olanzapine & $\begin{array}{l}\text { Aggression, bipolar disorders, manic episodes, } \\
\text { schizophrenia }\end{array}$ \\
\hline \multirow{4}{*}{$\begin{array}{r}\text { Antibody-drug } \\
\text { conjugate }\end{array}$} & Kadcyla $^{\mathrm{TM}}$ & Trastuzumab / emtansine & Metastatic breast cancer \\
\hline & Adcetris ${ }^{\circledR}$ & Brentuximab vedotin & $\begin{array}{l}\text { Hodgkin's Lymphoma, anaplastic large cell } \\
\text { lymphoma }\end{array}$ \\
\hline & Mylotarg® & Gentuzumab / Ozogamicin & Acute myeloid leukemia \\
\hline & Zevalin $^{\mathrm{TM}}$ & ${ }^{90} \mathrm{Y}$ & Non-Hodgkin's lymphoma \\
\hline \multirow{17}{*}{ Liposomes } & $\begin{array}{l}\text { Doxil }{ }^{\prime} / \\
\text { Caelyx } ®\end{array}$ & Doxorubicin & $\begin{array}{l}\text { Kaposi's Sarcoma, ovarian and breast cancer, } \\
\text { Multiple myeloma }\end{array}$ \\
\hline & Myocet ${ }^{\circledR}$ & Doxorubicin & $\begin{array}{l}\text { Combination therapy for metastatic breast } \\
\text { cancer }\end{array}$ \\
\hline & LipoDox $®$ & Doxorubicin & Kaposi' sarcoma, breast and ovarian cancer \\
\hline & Daunoxome ${ }^{\circledR}$ & Daunorubicin & Kaposi's sarcoma \\
\hline & DepoCyt ${ }^{\mathrm{TM}}$ & Cytarabine & Lymphomatous meningitis \\
\hline & Marqibo ${ }^{\circledR}$ & Vincristine & Acute lymphoblastic leukemia \\
\hline & Onco TCS $®$ & Vinciristine & Relapsed aggressive non-Hodgkin's lymphoma \\
\hline & Onivyde ${ }^{\circledR}$ & Irinotecan & Pancreatic cancer \\
\hline & Mepact & Mifamurtide & Osteosarcoma \\
\hline & Abelcet ${ }^{\circledR}$ & Amphotericin B & Severe fungal infections \\
\hline & Ambisome ${ }^{\circledR}$ & Amphotericin B & Fungal infections \\
\hline & Amphotec $\AA$ & Amphotericin B & Aspergillosis, visceral leishmaniasis \\
\hline & DoceAqualip $^{\mathrm{TM}}$ & Docetaxel & $\begin{array}{l}\text { Breas, gastric, head and neck and prostate } \\
\text { cancers }\end{array}$ \\
\hline & DepoDur ${ }^{\circledR}$ & Morphine & Postoperative pain \\
\hline & Epaxal® & Hepatitis A vaccine & Hepatitis A \\
\hline & Inflexal® V & Influenza virus vaccine & Influenza virus infections \\
\hline & Visudyne ${ }^{\circledR}$ & Verteporfin & $\begin{array}{l}\text { Age-related macular degeneration, choroidal } \\
\text { neovascularization }\end{array}$ \\
\hline \multirow{3}{*}{$\begin{array}{r}\text { Polymeric } \\
\text { Nanoparticles }\end{array}$} & $\begin{array}{l}\text { Zinostatin } \\
\text { stimalamer }\end{array}$ & Neocarzinostatin & Liver cancer \\
\hline & Lupron Depot® & Leuprolide & $\begin{array}{l}\text { Prostate, breast, ovarian and endometrial } \\
\text { cancers. Endometriosis, infertility, benign } \\
\text { prostatic hyperplasia }\end{array}$ \\
\hline & Abraxane $\AA$ & Paclitaxel & $\begin{array}{l}\text { Metastatic breast cancer, NSCLC, pancreatic, } \\
\text { ovarian, fallopian tubes and primary peritoneal } \\
\text { cancers }\end{array}$ \\
\hline $\begin{array}{r}\text { Polymeric } \\
\text { Micelles }\end{array}$ & Genexol®PM & Paclitaxel & $\begin{array}{l}\text { Metastatic or recurrent breast cancer, } \\
\text { Advanced or metastatic non-small cell lung } \\
\text { cancer (NSCLC) }\end{array}$ \\
\hline \multirow{4}{*}{$\begin{array}{r}\text { Polymer-Drug } \\
\text { conjugate }\end{array}$} & Oncaspar ${ }^{\circledR}$ & L-asparaginase & $\begin{array}{l}\text { Acute lymphoblastic leukemia, Non-Hodgkin's } \\
\text { lymphoma }\end{array}$ \\
\hline & Adagen ${ }^{\circledR}$ & Adenosine deaminase & Severe combined immunodeficiency (SCID) \\
\hline & Pegasys ${ }^{\circledR}$ & IFN- $\alpha-2 \mathrm{a}$ & Hepatitis B and C \\
\hline & CImzia ${ }^{\circledR}$ & Fab' fragment against $\mathrm{TNF} \alpha$ & $\begin{array}{l}\text { Active rheumatoid arthritis, axial } \\
\text { spondyloarthritis, ankylosing spondylitis, no- } \\
\text { radiographic axial spondyloarthritis and } \\
\text { psoriatic arthritis }\end{array}$ \\
\hline $\begin{array}{r}\text { Inorganic } \\
\text { Nanoparticles }\end{array}$ & NanoTherm® & Iron Oxide Nanoparticles & Glioblastoma \\
\hline
\end{tabular}

